\title{
Epoxyeicosatrienoates are the dominant eicosanoids in human lungs upon microbial
} challenge

\author{
L. Kiss*, H. Schütte" , W. Padberg", N. Weissmann+, K. Mayer*, T. Gessler*, \\ R. Voswinckel*, W. Seeger* and F. Grimminger*
}

ABSTRACT: Lipoxygenase, cyclo-oxygenase and cytochrome P450 (CYP) products of arachidonic acid (AA) are implicated in pulmonary vasoregulation. The CYP-mediated epoxyeicosatrienoates (EETs) have been described previously as the predominant eicosanoids in human lungs upon stimulation with the $\mathrm{Ca}^{2+}$ ionophore A23187. In this study, we challenged perfused human lungs with two microbial agents: Escherichia coli haemolysin (ECH) and formylmethionyl-leucyl-phenylalanine (fMLP).

Both stimuli elicited pronounced generation of leukotrienes (LTs), hydroxyeicosatetraenoic acids (HETEs), prostanoids (PTs) and EETs/dihydroxyeicosatrienoic acids (DHETs), as assessed by liquid chromatography-mass spectrometry, paralleled by pulmonary artery pressor response and lung oedema formation. The maximum buffer concentrations of EETs/DHETs surpassed those of LTs plus HETEs and PTs by a factor of four (ECH) or three (AA/fMLP). Dual 5-lipoxygenase/ cyclo-oxygenase inhibition caused pronounced reduction of AA/fMLP-induced LT/PT synthesis and oedema formation but only limited attenuation of pulmonary vasoconstriction, while inhibition of CYP epoxygenase clearly attenuated AA/fMLP-induced EET/DHET synthesis and vasoconstriction but not oedema formation, suggesting a major contribution of LTs/PTs to vascular leakage and of EETs/DHETs to pressor response.

Consequently, generation of EETs/DHETs is greater than that of LTs plus HETEs and PTs in ex vivo perfused human lungs upon microbial challenge suggesting a substantial contribution of these mediators to inflammatory-infectious pulmonary injury.

KEYWORDS: Cyclo-oxygenase, cytochrome P450, eicosanoids, human lung, lipoxygenase, soluble epoxide hydrolase

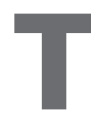

he lung vasculature is known to be a rich source of lipid mediator biosynthesis from arachidonic acid (AA) via the enzymatic pathways of the AA cascade: lipoxygenase (LOX), cytochrome P450 (CYP), soluble epoxide hydrolase (sEH) and cyclo-oxygenase (COX) [1-12]. The different eicosanoids encompass the LOXmediated leukotrienes (LTs) and lipoxins (LXs), the CYP-mediated epoxyeicosatrienoic acids (epoxyeicosatrienoates; EETs), the sEH-mediated dihydroxyeicosatrienoic acids (DHETs), the COXmediated prostanoids (PTs) (prostaglandins (PGs) and thromboxanes (TXs)) and the cis-trans conjugated hydroxyeicosatetraenoic acids (HETEs) mediated by LOX, CYP or COX (overview in fig. 1). They display a broad array of biological functions, ranging from proinflammatory and vasoconstrictive, to anti-inflammatory and vasodilatory properties $[1,4,5,11]$.
LTs are implicated in a variety of vascular abnormalities occurring under conditions of lung inflammation, such as pre- and post-capillary vasoconstriction and vascular leakage (cysteinylLTs) as well as leukocyte chemotaxis and activation $\left(\mathrm{LTB}_{4}\right)$, while HETEs may additionally affect leukocyte kinetics and lung fluid homeostasis [5, $6,13]$. Studies with polymorphonuclear neutrophils (PMNs) in vitro and in buffer-perfused rabbit lungs, which are known to harbour large quantities of resident neutrophils, monocytes and lymphocytes even after extensive rinsing [14, 15], demonstrated high yields of LT and HETE formation upon simultaneous application of free AA and inflammatory ligands, such as formylmethionyl-leucyl-phenylalanine (fMLP) or the bacterial toxin Escherichia coli haemolysin (ECH) $[8,16,17]$. This abundant mediator generation is a result of substantial AA liberation at sites of

\section{AFFILIATIONS}

*University of Gießen Lung Center (UGLC)/Dept of Internal Medicine, -Dept of Surgery,

+UGLC/Excellence Cluster

Cardiopulmonary System (ECCPS),

Justus Liebig University, Gießen, and

${ }^{\#}$ Dept of Internal Medicine/Infectious and Respiratory Diseases, CharitéUniversitätsmedizin Berlin, Berlin, Germany.

CORRESPONDENCE

L. Kiss

Biochemical Laboratory, University of Gießen Lung Center (UGLC), Dept of Internal Medicine

Justus Liebig University Gießen Paul Meimberg Straße 5

D-35392 Gießen

Germany

E-mail: ladislau.kiss@

innere.med.uni-giessen.de

Received:

Jan 022009

Accepted after revision:

March 282010

First published online:

April 082010

European Respiratory Journal

Print ISSN 0903-1936

Online ISSN 1399-3003 


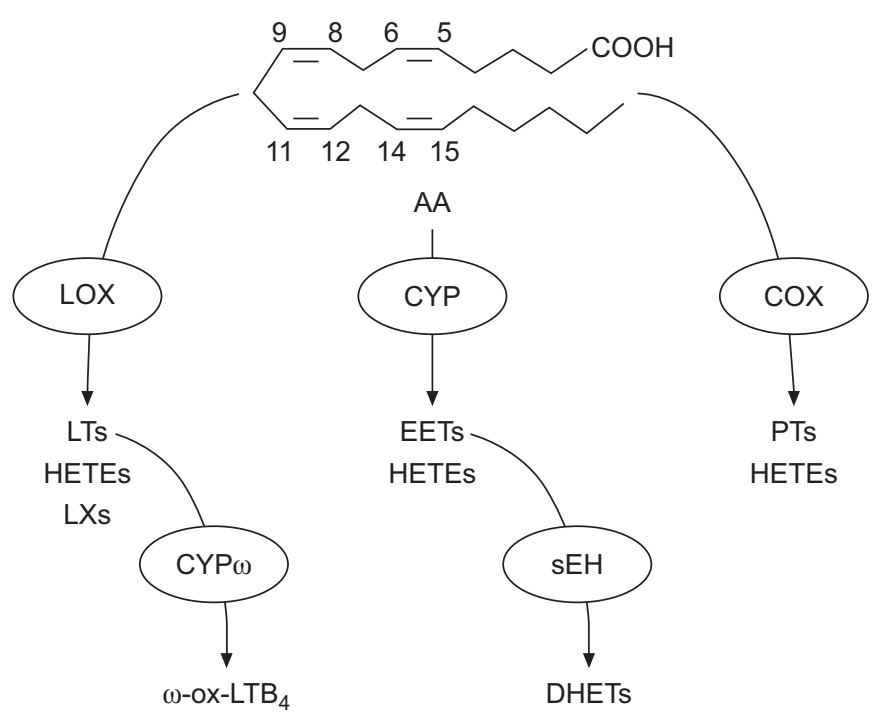

FIGURE 1. The main pathways of the arachindonic acid (AA) cascade. LOX lipoxygenase; $\mathrm{LT}$ : leukotrienes $\left(\mathrm{LTB}_{4}, \mathrm{LTC}_{4}, \mathrm{LTD}_{4}\right.$ and $\left.\mathrm{LTE} \mathrm{E}_{4}\right)$; HETE: hydroxyeicosatetraenoic acid (15-HETE, 11-HETE, 8-HETE, 12-HETE, 9-HETE and 5-HETE); LX lipoxin $\left(\mathrm{LXA}_{4}\right.$ and $\left.\mathrm{LXB}_{4}\right)$; CYP $\omega$ : cytochrome P450 $\omega$-oxygenase; $\omega$-ox-LTB 4 : $\omega$ oxygenase-mediated $\mathrm{LTB}_{4}$ metabolites (20-HO-LTB $4,20-\mathrm{COOH}-\mathrm{LTB}_{4}$ ); CYP: cytochrome P450; EETs: epoxyeicosatrienoic acids (14,15-EET, 11,12-EET, 8,9EET and 5,6-EET); sEH: soluble epoxide hydrolase; DHET: dihydroxyeicosatrienoic acid (14,15-DHET, 11,12-DHET, 8,9-DHET, 5,6-DHET and 5,6-DHET $\delta$-lactone); COX: cyclo-oxygenase; PT: prostanoids (prostaglandins $\left(\mathrm{PGl}_{2}, 6\right.$-keto- $\mathrm{PGF}_{1 \alpha}$, $\mathrm{PGE}_{2}, \mathrm{PGD}_{2}$ and $P \mathrm{PF}_{2 \alpha}$ ) and thromboxanes $\left(\mathrm{TXA}_{2}\right.$ and $\left.\mathrm{TXB}_{2}\right)$ ).

inflammatory events and leukocyte-endothelial cooperation in LT synthesis, including 1) a transfer of the unstable LT intermediate $\mathrm{LTA}_{4}$ from PMN feeder cells to acceptor endothelial cells (ECs) with subsequent employment of the enzymatic equipment of the latter for transformation into LTs, and 2) release of free AA from ECs with uptake and processing of this precursor by the adjacent PMNs [3, 16-21].

The ubiquitous, as yet poorly investigated, six HETE regioisomers display varying biological activities, ranging from anti-inflammatory (15-HETE) to proinflammatory (5-HETE) properties, and including chemotaxis (5-HETE, 11-HETE and 12-HETE) and regulation of the enzymatic pathways of the AA cascade $[1,5,13,22,23]$.

The physiological properties of the well-investigated PTs reach from vasoconstrictive $\left(\mathrm{TXA}_{2}, \mathrm{PGF}_{2 \alpha}\right)$ to vasodilatative $\left(\mathrm{PGI}_{2}\right.$, $\mathrm{PGD}_{2}$ and $\mathrm{PGE}_{2}$ ) functions $[1,5]$. TXA 2 and $\mathrm{PGI}_{2}$ represent a classical example of an antagonistic pair of mediators: vasocontriction and the resulting mismatch between pulmonary perfusion and ventilation provoked by $\mathrm{TXA}_{2}$ is counteracted by prostacyclin $\left(\mathrm{PGI}_{2}\right)$. Likewise, the adhesive and aggregatory actions of $\mathrm{TXA}_{2}$ are counteracted by the antiadhesive and antiaggregatory $\mathrm{PGI}_{2}$.

Among the AA-derived lipid mediators, the CYP-mediated EETs and their metabolites, the sEH-mediated DHETs, with reportedly contradictory vasoregulative functions, are of particular interest. They are described as both vasodilators and vasoconstrictors acting by themselves, via metabolisation by COX or by inducing the expression of COX and, thus, the enhanced production of prostanoids [11, 24-26]. In contrast to the systemic cardiovascular compartment, where EETs/ DHETs were proposed and discussed over a decade ago as chemical correlates of the vasodilatative endothelium-derived hyperpolarising factor (EDHF) [27-30], the role of these CYP/ $\mathrm{sEH}$-mediated autacoids in the pulmonary circulation is poorly investigated. Interestingly, first reports describe them as both vasoconstrictors and vasodilators [11, 24] and, as previously described by our group [10], inflammatory challenge of human lungs with the nonphysiological $\mathrm{Ca}^{2+}$ ionophore A23187 evoked the enhanced release of a broad array of eicosanoids, with the CYP-mediated EETs being by far the predominant mediators, paralleled by an increase in pulmonary arterial pressure $(\Delta P$ pa $)$ and lung weight gain $(\Delta W)$.

In the present study, we extended this approach by asking whether the challenge of human lungs with pathophysiologically relevant microbial stimuli per se, such as the proteinaceous transmembrane pore-forming exotoxin ECH and the inflammatory and chemotactic bacterial receptor-operated tripeptide fMLP, administered in the presence of exogenous AA availability might elicit a similar eicosanoid profile as A23187 and whether such metabolic responses are linked with vasoconstrictor response and oedema formation in the human pulmonary compartment. Studies were performed with isolated, perfused and ventilated intact human lungs, employing a combined technique of multistep solid-phase extraction (SPE) of buffer fluid followed by isocratic capillary liquid chromatography (CapLC) with dual online photodiode array detection (PDAD) and electrospray ionisation (ESI) tandem massspectrometric (MS/MS) detection for simultaneous assessment of all LOX-, COX-, CYP- and SEH-mediated representatives of the AA cascade [31]. Comprehensive eicosanoid profiling with this CapLC-PDAD-ESI-MS/MS method revealed the release of virtually all the main representatives of the AA cascade in response to both microbial challenges, with the absolute predominance (by a factor of three to four) of the CYP/sEHmediated EETs/DHETs over the other lipid mediators. This mediator liberation profile was paralleled by a pulmonary arterial vasoconstrictor response, to which the CYP epoxygenase pathway may particularly contribute, and $\Delta W$, which may be mainly a result of LOX and COX activity.

\section{MATERIALS AND METHODS}

\section{Materials}

AA, 20-carboxy-LTB 4 , 20-hydroxy-LTB ${ }_{4}, \mathrm{LTC}_{4}, \mathrm{LTD}_{4}, \mathrm{LTE}_{4}$, $\mathrm{PGB}_{1}, 6 \mathrm{t}-\mathrm{LTB}_{4}, 6 \mathrm{t}, 12 \mathrm{e}-\mathrm{LTB}_{4}, \mathrm{LTB}_{4}, \mathrm{LXA}_{4}, \mathrm{LXB}_{4}, 5 \mathrm{~S}, 6 \mathrm{R}-\mathrm{DiHETE}$, 5S,6S-DiHETE, and 15-, 11-, 8-, 12-, 9- and 5-HETE, were purchased from Biomol GmbH (Hamburg, Germany). 14,15-, 11,12-, 8,9-, 5,6-EET, and 14,15-, 11,12-, 8,9-, 5,6-DHET and 5,6DHET $\delta$-lactone were supplied by Biomol and by Cayman Chemical Company (Ann Arbor, MI, USA). 6-keto-PGF ${ }_{1 \alpha}$ $\mathrm{PGD}_{2}, \mathrm{PGE}_{2}, \mathrm{PGF}_{2 \alpha}, \mathrm{TXB}_{2}$ and stearidonic acid (SDA) were obtained from Cayman. fMLP, butylated hydroxytoluene (BHT), diethylamine (DEA) and triethylamine were obtained from Sigma Chemie GmbH (Munich, Germany). The 5-LOX inhibitor MK886, the COX inhibitor diclofenac and the CYP epoxygenase inhibitor $N$-methylsulfonyl-6-(2-propargyloxyphenyl)hexanamide (MS-PPOH) were from Cayman. EDTA disodium salt dihydrate, EDTA tetrasodium salt tetrahydrate $\left(\mathrm{Na}_{4} \mathrm{EDTA} \cdot 4 \mathrm{H}_{2} \mathrm{O}\right)$ and formic acid (FA) were purchased from 
E. Merck (Darmstadt, Germany). Water was purified with a Milli-Q system (Millipore, Eschborn, Germany). Methanol was purchased from Burdick and Jackson (Muskegon, MI, USA), acetonitrile was purchased from J.T. Baker (Deventer, The Netherlands) and isopropyl alcohol was obtained from Fluka AG (Buchs, Switzerland). Krebs-Henseleit buffer (KHB) was purchased from Serag Wiessner (Naila, Germany).

\section{Preparation of $\mathrm{ECH}$}

The exotoxin was kindly provided by S. Bhakdi (Institute of Medical Microbiology and Hygiene, Johannes Gutenberg University Mainz, Mainz, Germany) being prepared as previously described [32]. The endotoxin content of the preparation was reduced to $\sim 3 \mathrm{ng}$ LPS per $\mu \mathrm{g}$ protein. The haemolytic titre was assessed directly before use and is expressed in hemolytic units (HU) per $\mathrm{mL} ; 1 \mathrm{HU} \cdot \mathrm{mL}^{-1}$ corresponded to $\sim 100 \mathrm{ng}$ protein per $\mathrm{mL}$.

\section{Ex vivo human and rabbit lung perfusion and experimental protocol}

The technique of ex vivo lung perfusion and ventilation has been described previously by our group [33] and has now been adapted to whole human lungs originating from patients suffering from bronchial carcinoma undergoing lung resection. The study was approved by the University Ethics Committee (Justus Liebig Univesity, Gießen, Germany) and informed consent was obtained from each patient prior to surgery. The vascular bed of the resected lungs was immediately and extensively rinsed with $\mathrm{KHB}$. The carcinoma area was clamped; for washout of blood, the perfusate was initially not recirculated. The flow was slowly increased to $400 \mathrm{~mL} \cdot \mathrm{min}^{-1}$. Then lungs were placed in a temperature-equilibrated housing chamber at $37^{\circ} \mathrm{C}$, freely suspended from a force transducer for monitoring of lung weight and ventilated with $5.3 \% \mathrm{CO}_{2}, 21 \% \mathrm{O}_{2}, 73.7 \% \mathrm{~N}_{2}$ (tidal volume $500 \mathrm{~mL}$; frequency 12 breaths $\cdot \mathrm{min}^{-1}$; end-expiratory pressure $4 \mathrm{mmHg}$ ). After a steady state period of 20-30 min, the perfusate was exchanged and the lungs were recirculatingly perfused with a pulsatile flow rate of $600 \mathrm{~mL} \cdot \mathrm{min}^{-1}(500 \mathrm{~mL}$ total volume). $15 \mathrm{~min}$ later, $100 \mathrm{HU} \mathrm{ECH}$ (i.e. a subcytolitic dose of $\left.0.2 \mathrm{HU} \cdot \mathrm{mL}^{-1} ; \mathrm{n}=5\right)$ or fMLP $(20 \mu \mathrm{M}$ final concentration in buffer $)$ in the presence of AA (AA/fMLP, $n=5)$ or alone (control for the AA/fMPL experiments, $n=2$ ) was bolus-injected into the pulmonary artery and time was set to zero. AA $(10 \mu \mathrm{M}$ final concentration) was added to the buffer $30 \mathrm{~s}$ before bolus injection of fMLP. Time-matched baseline control experiments $(n=2)$ were performed without application of $\mathrm{ECH}$ or (AA/)fMLP. In experiments with AA/fMLP challenge under conditions of simultaneous inhibition of 5-LOX and COX (10 $\mu \mathrm{M}$ MK886 and $30 \mu \mathrm{M}$ diclofenac, respectively; $\mathrm{n}=2$ ) or inhibition of CYP epoxygenase (10 $\mu \mathrm{M}$ MS-PPOH, $n=2)$, the respective inhibitors were added immediately after starting the recirculating perfusion. 10-mL perfusate samples were collected for eicosanoid analysis from the venous effluent $15 \mathrm{~min}$ before $(-15 \mathrm{~min})$, immediately prior to (0 min), as well as 2, 3, 5, 10, 15, 20, 30 and 45 min after AA/fMLP or ECH challenge. Eicosanoid concentration results were corrected for the progressive mediator concentrating effect resulting from the gradual reduction of the total perfusate volume due to removal of $10-\mathrm{mL}$ samples, by considering the total amount of each eicosanoid measured at each time-point as that contained in the initial total perfusate volume of $500 \mathrm{~mL}$. In addition, eicosanoid release levels and weight gain were corrected for differences in original lung weights by using one of the lungs as a reference lung for normalisation of the eicosanoid concentrations and $\Delta W$ for the other lungs.

The effect of exogenously supplied EET on pressor response and oedema formation was investigated using isolated, perfused and ventilated human $(n=2)$ and rabbit $(n=5)$ lungs employing the aforementioned experimental set-up. Rabbit lungs were perfused as described previously [33], with a flow rate of $150 \mathrm{~mL} \cdot \mathrm{min}^{-1}$ (250 $\mathrm{mL}$ total volume) and ventilated with the same gas mixture as human lungs. With both human and rabbit lungs, after the initial 15-min steady state period, 11,12-EET ( $0.3 \mu \mathrm{M}$ final concentration) was bolus-injected into the pulmonary artery and the time was set to zero.

\section{SPE and CapLC-PDAD-ESI-MS/MS equipment and procedures}

Eicosanoids were extracted from lung perfusate by adapting a previous SPE procedure [34] for the extraction of all representative mediators of the AA cascade. Briefly, after collection, all samples were supplemented with constant amounts of $\mathrm{PGB}_{1}$ and SDA as internal standards and reference substances for the calculation of the relative retention index (RRI), BHT as an antioxidant, $\mathrm{Na}_{4}$ EDTA $44 \mathrm{H}_{2} \mathrm{O}, \mathrm{FA}$ and isopropyl alcohol, and were vortexed, centrifuged and subjected to SPE employing octadecylsilane (ODS) cartridges (Chromabond C18ec, 200 mg sorbent mass; Macherey-Nagel, Düren, Germany). The extracted eicosanoids were subjected to CapLC-PDAD-ESI-MS/MS analysis, as described previously [31], employing a 1100 Series capillary LC unit (Agilent Technologies Deutschland $\mathrm{GmbH}$, Waldbronn, Germany) interfaced with an Esquire 3000+ ion trap mass spectrometer (Bruker Daltonik GmbH, Bremen, Germany). Separation of autacoids was performed on capillary columns (length $\times$ internal diameter $250 \mathrm{~mm} \times 300 \mu \mathrm{m}$; GROM-SIL 80 ODS-7 $\mathrm{pH}$ ) from Grom Analytik + HPLC GmbH (RottenburgHailfingen, Germany) employing as the mobile phase a mixture of water/acetonitrile/methanol/FA (130/240/80/ 0.22 , vol/vol/vol/vol; $\mathrm{pH} 4.30$, adjusted with DEA) at a flow rate of $2.7 \mu \mathrm{L} \cdot \mathrm{min}^{-1}$. The eluted compounds were subjected to dual online spectrophotometric and MS/MS analysis in negative-ion mode. Identity and purity of eluted eicosanoids were triple-checked: chromatographically by isocratic RRI as well as by dual ultraviolet spectrometric and MS/MS analysis.

\section{Statistics}

Analytical data, maximal $\Delta P$ pa $(\Delta P$ pa,max $)$ and maximal $\Delta W$ $(\Delta W$ max $)$ are expressed as mean \pm SEM. Statistical evaluation was performed using one-way ANOVA. A p-value $<0.05$ was considered statistically significant.

\section{RESULTS}

\section{Overview of lipid mediator generation}

Both challenge with $\mathrm{ECH}$ and combined application of the precursor fatty acid (AA) and AMLP resulted in the liberation of all representative AA-derived LOX, CYP, sEH and COX products in the perfused human lungs. The following eicosanoid groups of the AA cascade (fig. 1) were analysed. 1) LOX-mediated LTs and LXs: $\mathrm{LTB}_{4}$, cysteinyl-LTs $\left(\mathrm{LTC}_{4}\right.$, $\left.\mathrm{LTD}_{4}, \mathrm{LTE}_{4}\right)$, $\omega$-oxidation metabolites of $\mathrm{LTB}_{4}\left(20-\mathrm{HO}-\mathrm{LTB}_{4}\right.$ and $20-\mathrm{COOH}-\mathrm{LTB}_{4}$ ) summarised as $\omega$-ox-LTB ${ }_{4}, \mathrm{LXA}_{4}$ and 
$\mathrm{LXB}_{4}$; 2) CYP epoxygenase-mediated oxiranes 14,15-EET, 11,12-EET, 8,9-EET and 5,6-EET; 3) sEH-mediated EET-derived vic-diols 14,15-DHET, 11,12-DHET, 8,9-DHET and 5,6-DHET; 4) COX-mediated PTs: 6-keto-PGF ${ }_{1 \alpha}$ as an indicator of the unstable prostacyclin $\left(\mathrm{PGI}_{2}\right), \mathrm{PGD}_{2}, \mathrm{PGE}_{2}, \mathrm{PGF}_{2 \alpha}$, and $\mathrm{TXB}_{2}$ as an indicator of the unstable TXA $\mathrm{T}_{2}$; and 5) LOX-, CYP- or COXmediated 15-HETE, 12-HETE, 11-HETE, 9-HETE, 8-HETE and 5-HETE.

The measured eicosanoid levels are absolute values at each time point, reflecting the specific dynamic equilibrium between release and reuptake or breakdown of each mediator and thus, depending on the velocity of the elimination process, they more or less accurately reflect actual liberation. According to the rate of removal after reaching maximal concentration, two types of patterns were observed: 1) avid elimination (generation/release clearly dominated by re-uptake/breakdown) with levels tending to zero for $\mathrm{LT}(\mathrm{B}, \mathrm{C}, \mathrm{D})_{4}$, PTs, (5-,8-,9-,12-)HETE and 2) minimal elimination (balance between generation/release and re-uptake/breakdown) leading to virtually plateauing levels for $\mathrm{LTE}_{4}, \omega$-ox-LTB 4 and 15-HETE and EETs/DHETs.
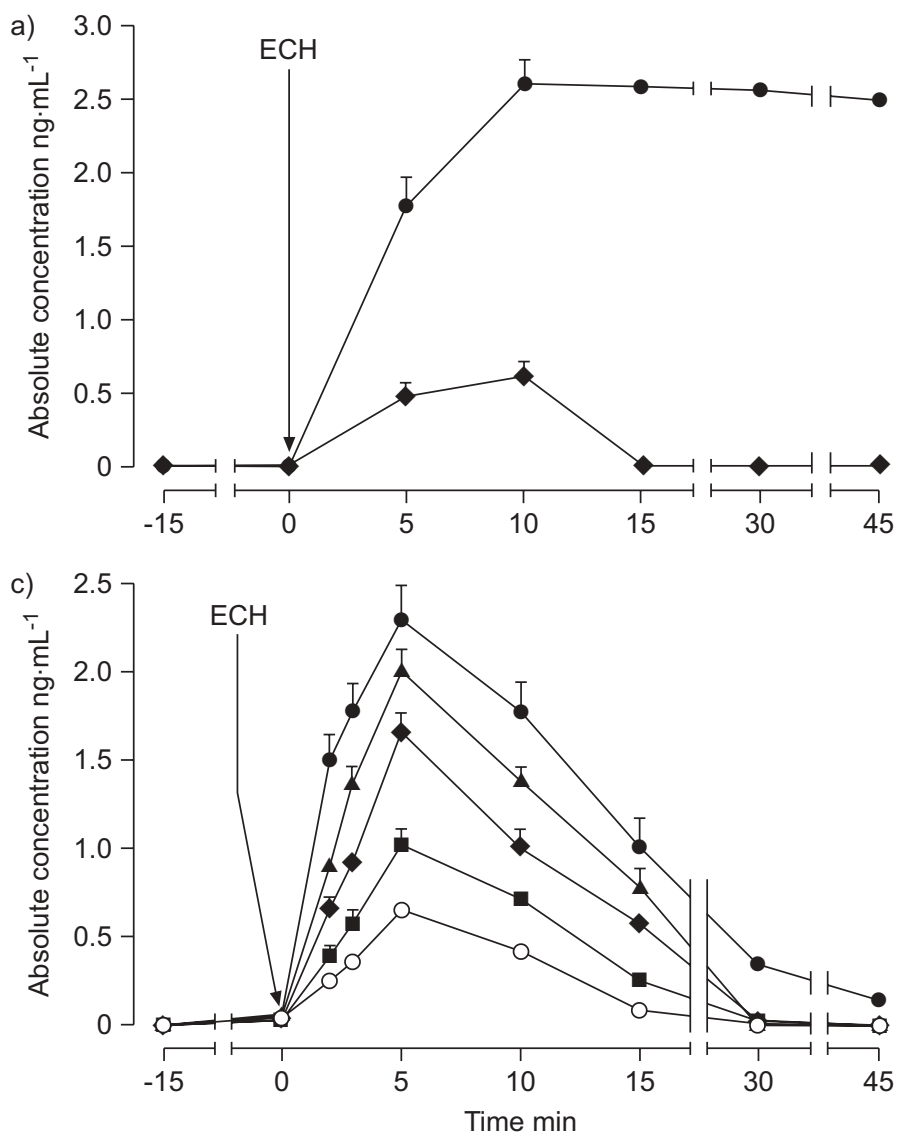

\section{Mediator release under baseline conditions}

In the initial 15-min baseline periods, i.e. in the absence of $\mathrm{ECH}$ or AA/fMLP administration, no baseline release of LTs (figs 2a and $3 \mathrm{a}$, respectively) and LXs (data not shown), but some moderate liberation of 15-HETE greater than that of (8-HETE + 12-HETE), 11-HETE, 9-HETE and 5-HETE individually was detected (figs $2 b$ and $3 b$ ). Virtually no baseline release of PTs was registered (figs $2 \mathrm{c}$ and $3 \mathrm{c}$ ). Substantial baseline liberation of 8,9-(EET+DHET) and 11,12-(EET+DHET) was noted, in contrast to only moderate release of 5,6-(EET+DHET) and 14,15-(EET+DHET) (figs $2 \mathrm{~d}$ and $3 \mathrm{~d}$ ). In the time-matched baseline controls without inflammatory challenge, moderate liberation of HETEs, more substantial appearance of EETs/ DHETs but no LT, PT (fig. 4a) and LX (data not shown) release was registered.

\section{$L T$ and $L X$ release upon ECH challenge}

Administration of $\mathrm{ECH}$ provoked rapid release of $\mathrm{LTB}_{4}$ peaking after $10 \mathrm{~min}$, and enhanced liberation with subsequent plateauing of $\mathrm{LTE}_{4}$. The maximum perfusate level of $\mathrm{LTE}_{4}$ surpassed that of $\mathrm{LTB}_{4}$ four-fold (fig. 2a). No $\mathrm{LTC}_{4}, \mathrm{LTD}_{4}$,
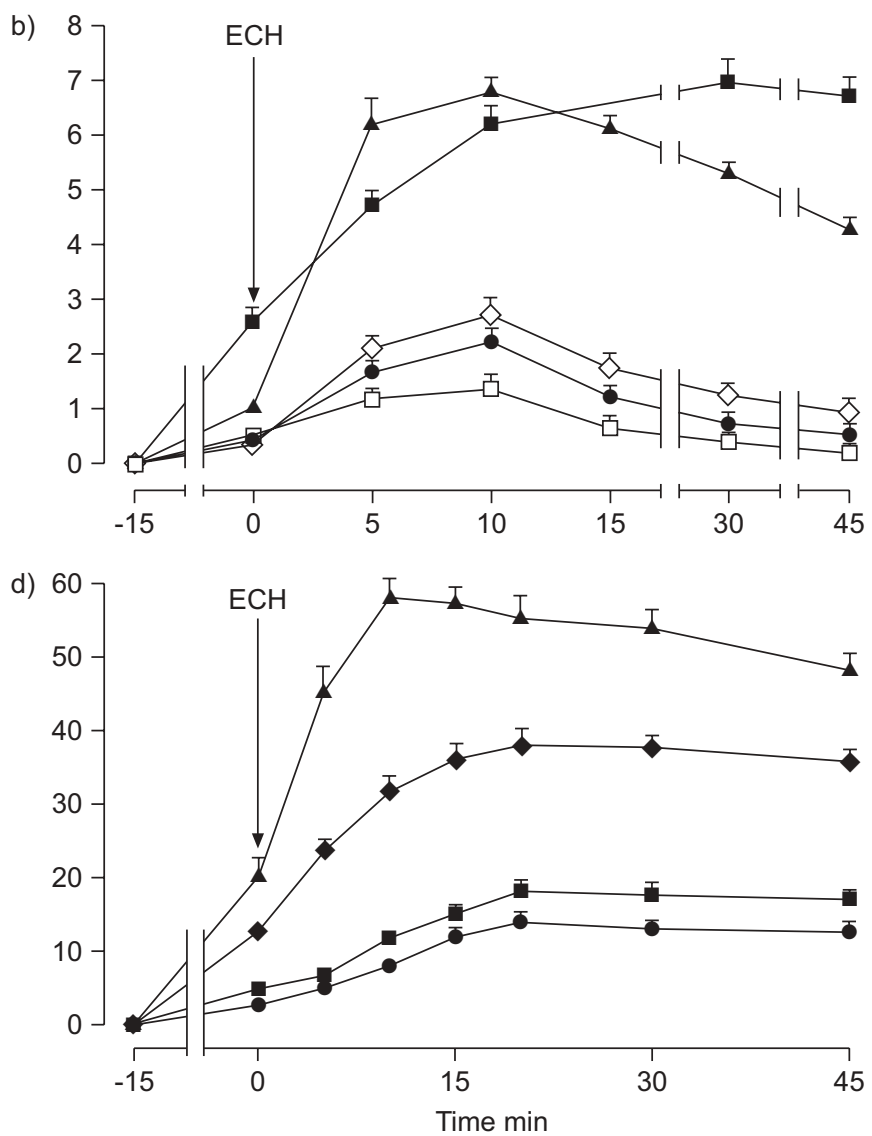

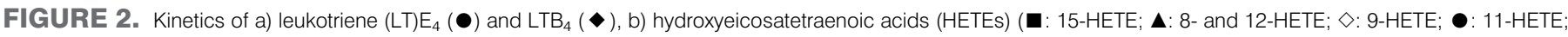

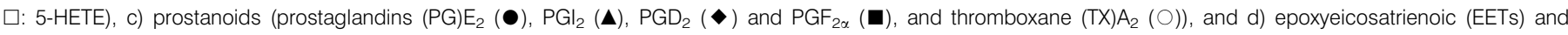

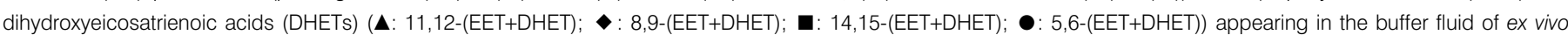

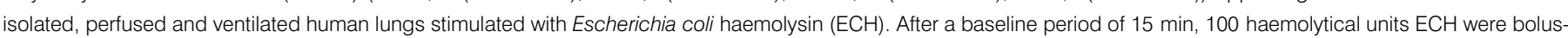

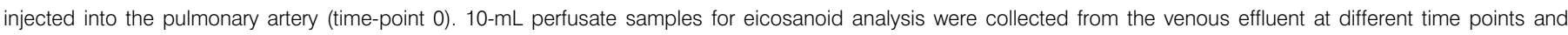

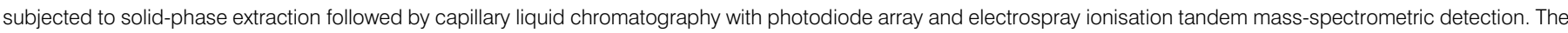

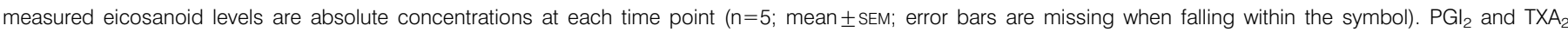
concentrations were calculated from the measured 6-keto-PGF ${ }_{1 \alpha}$ and $\mathrm{TXB}_{2}$ concentrations, respectively. 

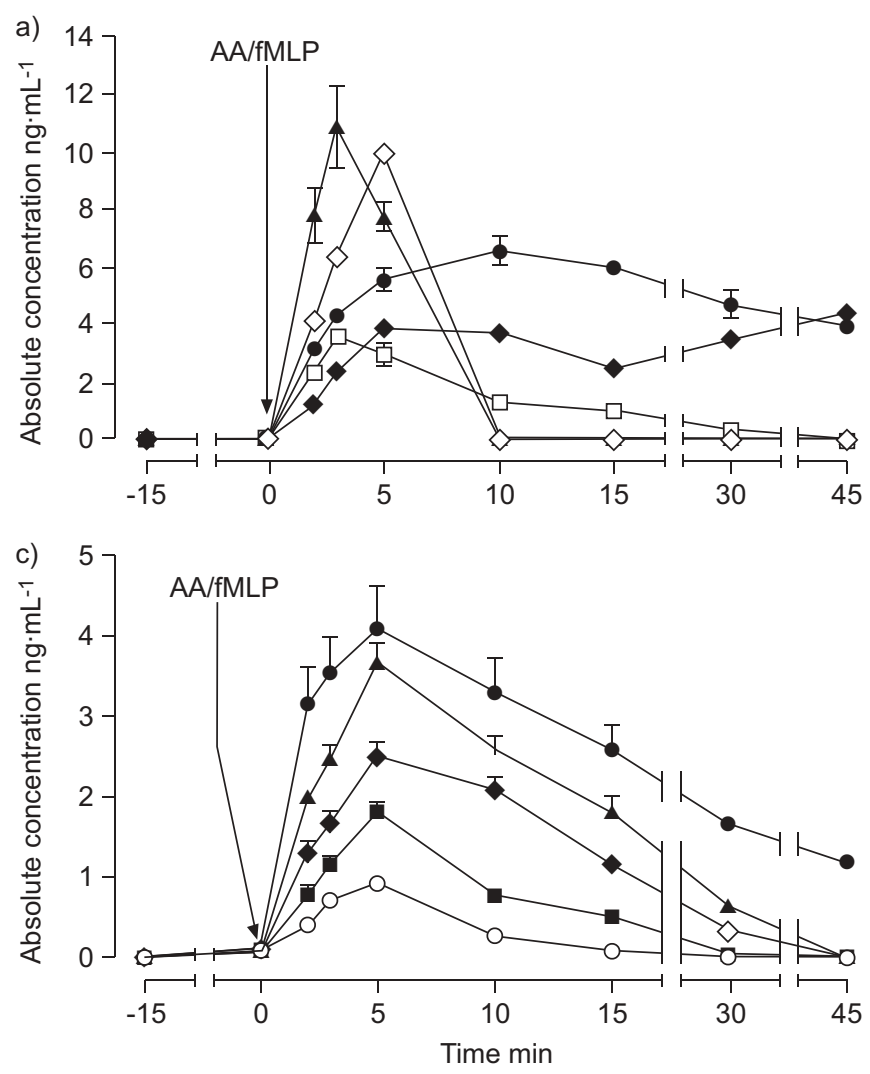

b)

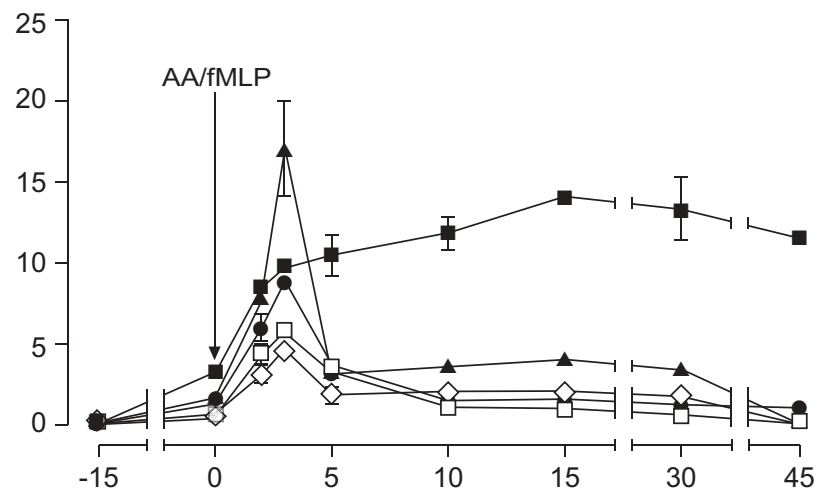

d) 200

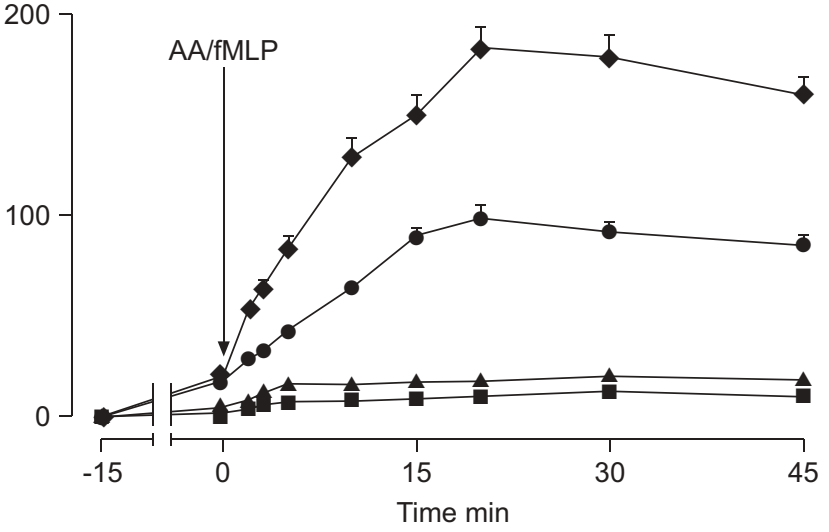

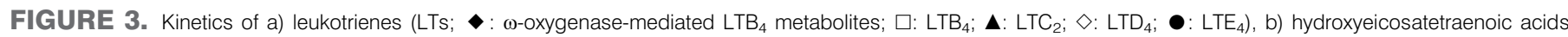

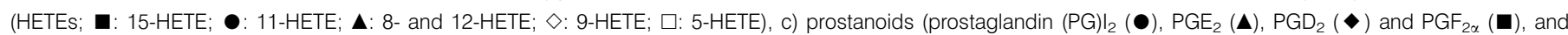
thromboxane $\left.(\mathrm{TX}) \mathrm{A}_{2}(\mathrm{O})\right)$, and d) epoxyeicosatrienoic (EETs) and dihydroxyeicosatrienoic acids (DHETs) $\bullet:$ : 8,9-(EET+DHET); $:$ : 11,12-(EET+DHET); $\mathbf{\Delta}: 5,6-(\mathrm{EET}+\mathrm{DHET})$; - : 14,15-(EET+DHET)) released in the buffer of isolated, perfused and ventilated human lungs challenged with arachidonic acid/formyl-methionyl-leucyl-phenylalanine (AA/ fMLP). After a 15-min baseline period, lungs were challenged with AA and fMLP (20 $\mu \mathrm{M}$ and $10 \mu \mathrm{M}$ final concentration, respectively; time-point 0). 10-mL perfusate samples were collected from the venous effluent at different time points and subjected to solid-phase extraction followed by capillary liquid chromatography with photodiode array and electrospray ionisation tandem mass-spectrometric detection. The measured eicosanoid levels are absolute concentrations at each time point ( $n=5$; mean \pm SEM; error bars are missing when falling within the symbol). $\mathrm{PGI}_{2}$ and $\mathrm{TXA}_{2}$ concentrations were calculated from the measured 6-keto-PGF ${ }_{1 \alpha}$ and $\mathrm{TXB}_{2}$ concentrations, respectively.

$\mathrm{LXA}_{4}$ or $\mathrm{LXB}_{4}$ was detected. Virtually no $(<1 \%)$ hydrolytic decay products $\left(6 \mathrm{t}-\mathrm{LTB}_{4}, 6 \mathrm{t}, 12 \mathrm{e}-\mathrm{LTB}_{4}, 5 \mathrm{~S}, 6 \mathrm{R}\right.$-DiHETE or $5 \mathrm{~S}, 6 \mathrm{~S}$ DiHETE) of the unstable LT intermediate $\mathrm{LTA}_{4}$ were detected.

\section{HETE liberation after ECH challenge}

After moderate baseline release, $\mathrm{ECH}$ induced peaking kinetics of 8- and 12-HETE, paralleled by enhanced liberation and subsequent plateauing of 15 -HETE, with $c \max \left(6.76 \mathrm{ng} \cdot \mathrm{mL}^{-1}\right.$ and $6.95 \mathrm{ng} \cdot \mathrm{mL}^{-1}$, respectively) by far higher than those of 5-, 9- and 11-HETE, which displayed kinetics comparable to 8and 12-HETE (fig. 2b).

\section{PT formation elicited by administration of $\mathrm{ECH}$}

Administration of ECH provoked the liberation of all PTs with comparable kinetics, at concentrations of $\sim 0.5-2.5 \mathrm{ng} \cdot \mathrm{mL}^{-1}$, peaking after $5 \mathrm{~min}$ (fig. 2c).

\section{EET/DHET generation provoked by ECH challenge}

ECH elicited the release of all EETs/DHETs, with a rank order of 11,12-(EET+DHET) $>8,9-($ EET+DHET) $>14,15-($ EET+DHET) $>5,6-($ EET+DHET) (fig. 2d). 11,12-(EET+DHET) and 8,9(EET+DHET) displayed rapidly increasing liberation profiles reaching by far higher $c \max \left(57.90 \mathrm{ng} \cdot \mathrm{mL}^{-1}\right.$ and $37.50 \mathrm{ng} \cdot \mathrm{mL}^{-1}$, respectively) than 14,15-(EET+DHET), 5,6-(EET+DHET) and all other eicosanoid classes. The sum of the quantitatively dominating regioisomers 8,9-(EET+DHET) and 11,12-(EET+DHET) surpassed the sum of the minor regioisomers 5,6-(EET+DHET) and 14,15-(EET+DHET) by a factor of three. The total amount of EETs/ DHETs surpassed the sum of LTs, HETEs and PTs four-fold (table 1, fig. 4a).

\section{Eicosanoid release upon challenge with fMLP alone}

In the experiments with fMLP alone, moderate liberation of HETEs and more substantial appearance of EETs/DHETs, but no LT, PT (fig. 4a) or LX (data not shown) release was registered.

\section{$L T$ and $L X$ liberation after coapplication of $A A$ and $f M L P$}

$\mathrm{AA} / \mathrm{fMLP}$ provoked the rapid appearance of $\mathrm{LTB}_{4}$, $\omega$-ox-LTB (20-HO-LTB 4 and 20-COOH-LTB ${ }_{4}$ ) and the cysteinyl-LTs, $\mathrm{LT}(\mathrm{C}, \mathrm{D}, \mathrm{E})_{4}$, in the perfusate (fig. 3a). In contrast, virtually no $(<1 \%)$ nonenzymatic $\mathrm{LTA}_{4}$ hydrolysis products were detected. Of the LTs, $\mathrm{LTC}_{4}$ and $\mathrm{LTD}_{4}$ displayed the highest peak values (10.82 ng. $\mathrm{mL}^{-1}$ and $9.92 \mathrm{ng} \cdot \mathrm{mL}^{-1}$, respectively; table 1$)$, followed by rapid decline, concomitant with progressive increase and subsequent plateauing of their metabolite $\mathrm{LTE}_{4}$. In 

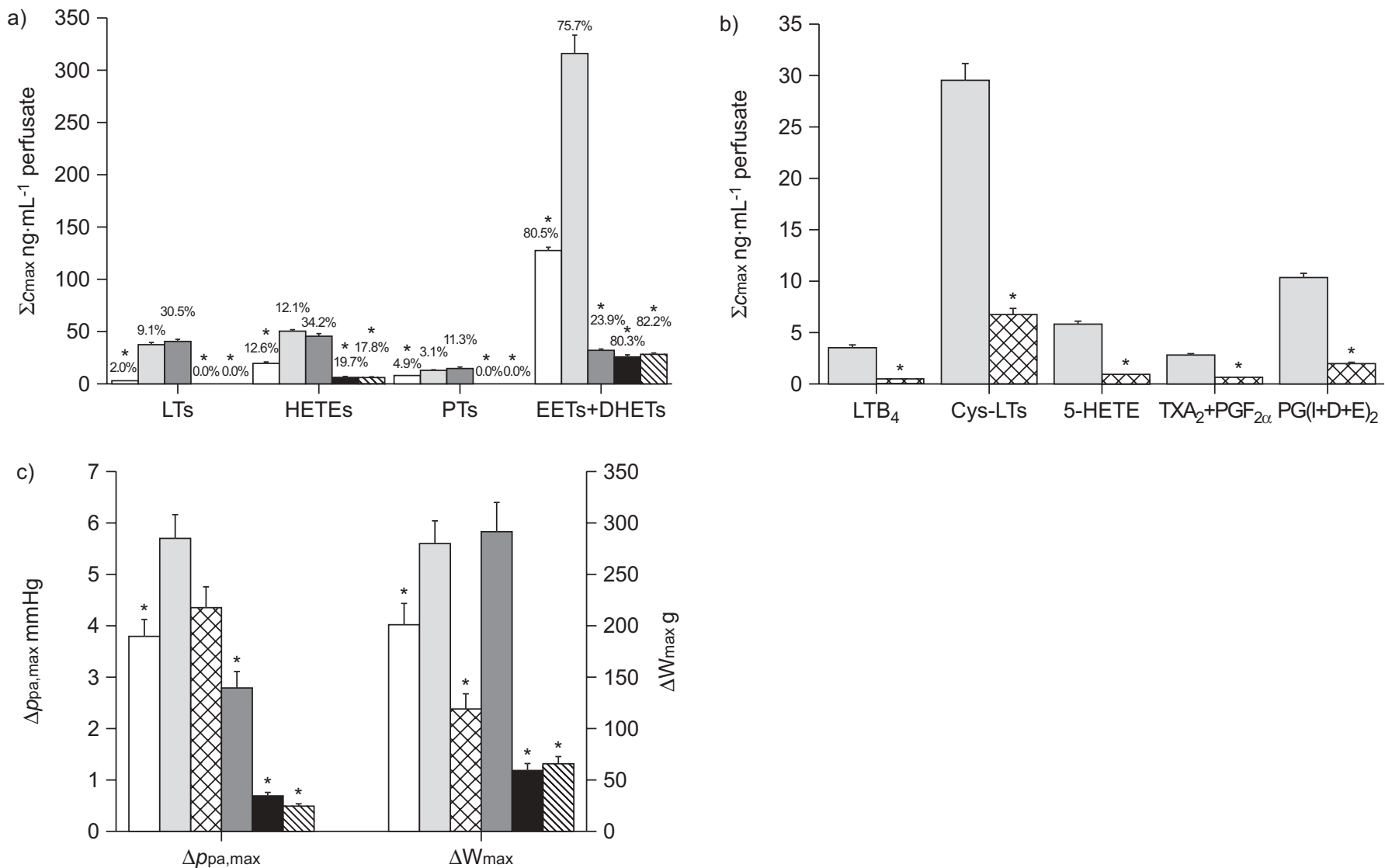

FIGURE 4. Comparison of the $a$ and b) metabolic (eicosanoid generation) and c) pathophysiological (vasoconstriction and oedema formation) response in isolated perfused human lungs under different conditions: challenge with Escherichia coli haemolysin $(E C H ; n=5 ; \square)$, arachidonic acid/formyl-methionyl-leucyl-phenylalanine (AA/ fMLP; $n=5 ;-1), A A / f M L P$ in the presence of both the lipoxygenase (LOX) inhibitor MK886 and the cyclooxygenase (COX) inhibitor diclofenac ( $n=2 ;$ presence of the selective cytochrome P450 epoxygenase inhibitor N-methylsulfonyl-6-(2-proargyloxyphenyl)hexanamide (MS-PPOH; n=2; $\mathbf{n})$, fMLP alone ( $\mathrm{n}=2$; $\mathbf{\square}$ ), and time-matched baseline control $(n=2 ; \mathbb{\$})$. a) The generation of the four main lipid mediator classes of the AA cascade, leukotrienes (LTs), hydroxyeicosatetraenoic acids (HETEs), prostanoids (PTs), and epoxyeicosatrienoic (EETs) and dihydroxyeicosatrienoic acids (DHETs) is presented as the sums of the means of the maximal perfusate concentrations ( $\Sigma c \max )$ of the eicosanoids belonging to the respective class. The values reported on top of the bars represent the relative amount of each eicosanoid class (LTs, HETEs, PTs, EETs and DHETs) as \% $\Sigma$ cmax as compared with the total amount of eicosanoids generated under the same experimental conditions (challenge with ECH, AA/fMLP, MS-PPOH+AA/fMLP, fMLP alone, or time-matched baseline control, respectively). The sum of percentages under the same experimental conditions amounts to $100 \%$. b) The reduction of $A A / f M L P$-induced synthesis of $L T B_{4}$, cysteinyl-LTs, 5-HETE, thromboxane $(T X) A_{2}$ plus $P G F_{2 \alpha}$, and $P G l_{2}$ plus $P G D_{2}$, $P G E_{2}$ resulting from dual inhibition of LOX and COX by MK886 and diclofenac, respectively. c) Inflammatory pulmonary vasoconstrictor response and endothelial permeability increase/oedema formation are expressed as maximal pulmonary arterial pressure rise ( $\triangle P$ pa,max) and maximal weight gain ( $\triangle W_{\text {max }}$ ), respectively. Data are presented as mean \pm SEM; error bars are missing when falling within the bar. *: $p<0.05$ versus challenge with AA/fMLP.

addition, rapid peaking of $\mathrm{LTB}_{4}$ was noted (maximum values approximately one-third of those for $\mathrm{LTC}_{4}$ ), with subsequent plateauing of its $\omega$-oxidation products. The total amount (measured as by the sum of cmax values) of LTs was one order of magnitude above that elicited by application of $\mathrm{ECH}$ (table 1). No $\mathrm{LXA}_{4}$ or $\mathrm{LXB}_{4}$ was detected.

\section{HETE release elicited by administration of AA/fMLP}

Following baseline release, AA/fMLP provoked enhanced release of all HETEs. The highest perfusate concentration was noted for 8-HETE and 12-HETE, peaking after $3 \mathrm{~min}(\mathrm{cmax}$ $17.07 \mathrm{ng} \cdot \mathrm{mL}^{-1}$ ), with subsequent rapid decline. 5-, 9- and 11HETE displayed kinetics comparable to 8- and 12-HETE, however, with lower peak values (fig. $3 b$ ). In contrast, enhanced liberation of 15-HETE resulted in markedly increased plateau levels reaching a maximum concentration of $14.06 \mathrm{ng} \cdot \mathrm{mL}^{-1}$. The total quantity of HETEs was three times higher than in ECH-challenged lungs (table 1).

\section{PT generation after AA/fMLP challenge}

Upon exposure to AA/fMLP, PTs showed generation profiles similar to those in ECH-challenged lungs: rapid peaking appearance followed by sustained decline of $\mathrm{PGI}_{2}$ (measured as the longer-lived 6-keto- $\left.\mathrm{PGF}_{1 \alpha}\right), \mathrm{PGD}_{2}, \mathrm{PGE}_{2}, \mathrm{PGF}_{2 \alpha}$ and $\mathrm{TXA}_{2}$ (measured as the longer-lived $\mathrm{TXB}_{2}$ ) was registered (fig. 3c). The highest peak values were noted for $\mathrm{PGI}_{2}$ and $\mathrm{PGE}_{2}$ (4.11 ng. $\mathrm{mL}^{-1}$ and $3.68 \mathrm{ng} \cdot \mathrm{mL}^{-1}$, respectively). The total PT quantity was 1.8-times higher than upon $\mathrm{ECH}$ challenge.

\section{EET/DHET formation elicited by AA/fMLP challenge}

AA/fMLP challenge provoked dramatically enhanced and progressive 8,9-(EET+DHET) release (fig. $3 \mathrm{~d})$, displaying by 


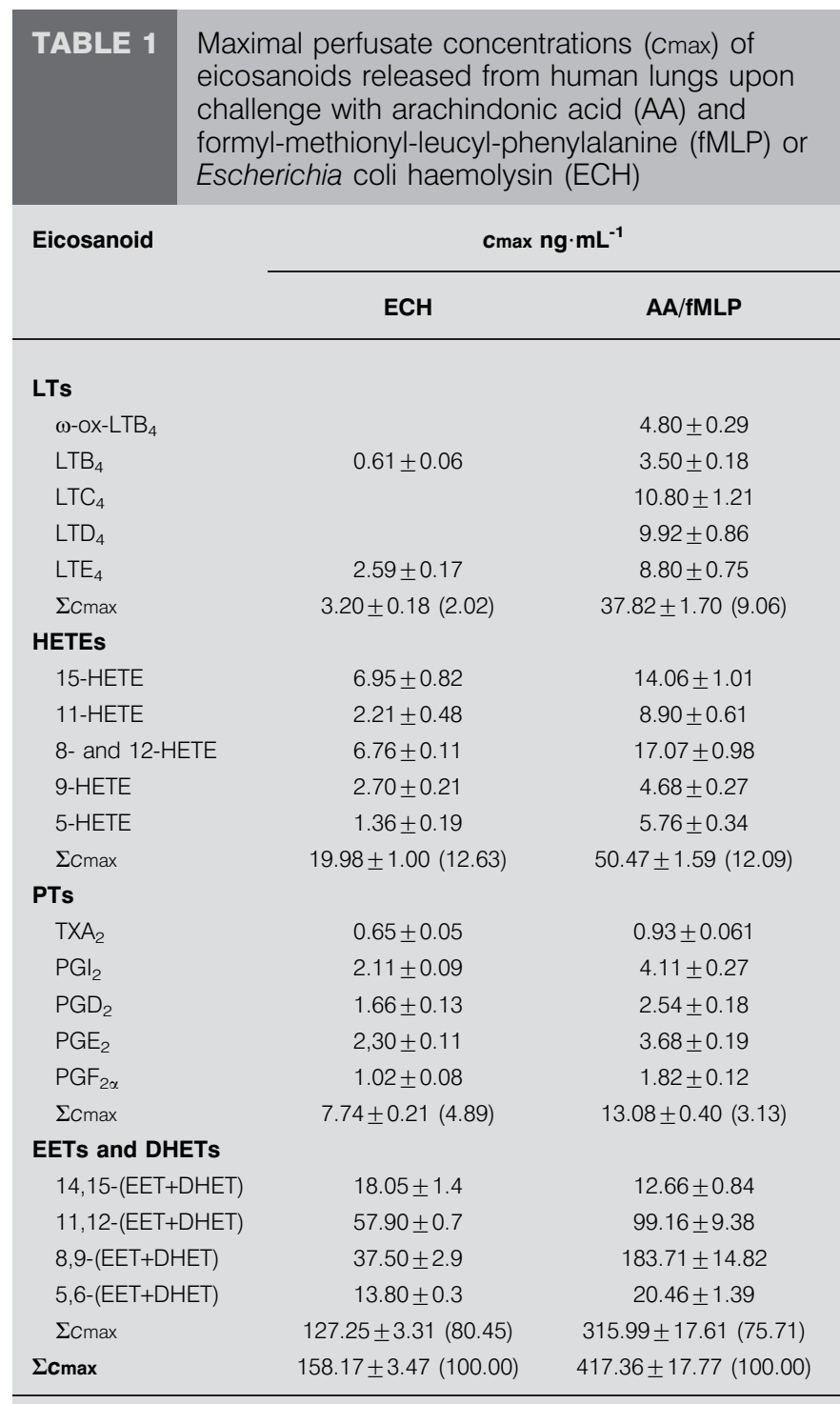

Data are presented as mean \pm SEM or mean $\pm \operatorname{SEM}(\%) . n=5$. LT: leukotriene; $\omega$-ox-LTB ${ }_{4}$ : $\omega$-oxygenase-mediated $\mathrm{LTB}_{4}$ metabolites; $\Sigma \mathrm{Cmax}$ : sum of $\mathrm{cmax}$ values; HETE: hydroxyeicosatetraenoic acid; PT: prostanoid; TX: thromboxane; PG: prostaglandin; EET: epoxyeicosatrienoic acid; DHET: dihydroxyeicosatrienoic acid.

far the highest maximal perfusate concentration of all eicosanoids analysed in our study $\left(c \max 183.71 \mathrm{ng} \cdot \mathrm{mL}^{-1}\right.$; table 1). 11,12-(EET+DHET) showed a similar profile, reaching half the $c$ max of 8,9-(EET+DHET). In addition, AA/fMLP induced some minor liberation of 14,15-(EET+DHET) and 5,6(EET+DHET). The sum of the quantitatively major regioisomers 8,9-(EET+DHET) and 11,12-(EET+DHET) surpassed the sum of the minor regioisomers 5,6-(EET+DHET) and 14,15(EET+DHET) by a factor of 8.5. The total quantity of the EETs / DHETs surpassed the total sum of all LTs, HETEs and PTs three-fold (table 1 and fig. 4a). Notably, the 8,9-(EET+ DHET) $>11,12-($ EET+DHET) predominance found in response to AA/fMLP stimulation differed from the 11,12-(EET+ DHET) $>8,9-(E E T+D H E T)$ predominance in response to $\mathrm{ECH}$ challenge. The overall amount of EETs/DHETs post AA/fMLP was 2.6-times higher than post-ECH.

\section{Eicosanoid release upon AA/fMLP challenge after previous CYP, LOX and COX inhibition}

AA/fMLP challenge of human lungs after previous administration of the CYP epoxygenase inhibitor MS-PPOH resulted in a dramatic attenuation of EET/DHET release, while the liberation of the other eicosanoid groups (LTs, HETEs and PTs) was not significantly affected (fig. 4a).

Dual inhibition of 5-LOX and COX in AA/fMLP-challenged human lungs with MK886 and diclofenac, respectively, resulted in marked decrease in LT/5-HETE and PT generation (fig. 4b), while no significant change in the appearance of 8-, 9-, 11-, 12- and 15-HETE and EETs/DHETs was registered (data not shown).

\section{Pulmonary vascular response provoked by $E C H, A A / f M L P$ and EET challenge}

Near-constant $P_{\text {pa }}(9-10 \mathrm{mmHg})$ was noted in the initial 15min baseline periods, as well as in the time-matched baseline control experiments and in the control experiments with fMLP alone $(\Delta P$ pa $<1 \mathrm{mmHg}$; fig. $4 \mathrm{c})$. Challenge with both $\mathrm{AA} /$ fMLP and ECH provoked a pronounced vascular pressor response with $\Delta P$ pa,max of $5.7 \pm 0.47 \mathrm{mmHg}$ and $3.8 \pm$ $0.32 \mathrm{mmHg}$, respectively, accompanied by marked oedema formation resulting in a $\Delta W \max$ of $280.1 \pm 22.1 \mathrm{~g}$ and $201.1 \pm$ $21.1 \mathrm{~g}$, respectively. In experiments with AA/fMLP-challenged human lungs, simultaneous inhibition of LT and PT synthesis with MK886 and diclofenac, respectively, resulted in marked reduction of $\Delta W \max$ but only limited attenuation of $\Delta P$ pa,max as compared with AA/fMLP challenge without inhibitors, while blockade of EET/DHET generation with MS-PPOH was paralleled by substantial attenuation of $\Delta P$ pa,max but not of $\Delta W_{\max }$.

Application of exogenous 11,12-EET to human $(n=2)$ and rabbit $(n=5)$ lungs in approximately the same concentration as that of the endogenously released 11,12-EET $(0.3 \mu \mathrm{M})$, provoked marked $\Delta P$ pa,max $-3.1 \pm 0.27 \mathrm{mmHg}$ and $3.4 \pm 0.31 \mathrm{mmHg}$ with human and rabbit lungs, respectively, but no oedema formation: $\Delta W<75 \mathrm{~g}$ with human lungs, i.e. the same range as in the timematched baseline control experiments (fig. 4c) and $\Delta W<1 \mathrm{~g}$ with rabbit lungs (data not shown).

\section{DISCUSSION}

Previous investigations performed with non-human lungs of different species under various pathophysiological conditions, including challenge with A23187, ECH and fMLP [2, 3, 5, 8, 9, $18,19,31,35-42]$, revealed the generation of individual eicosanoids/eicosanoid groups (TXA $2, \mathrm{PGI}_{2}, \mathrm{LT}$ s or HETEs), paralleled by pressor response and increased endothelial permeability. Intriguingly, challenge of human lungs with A23187 elicited the generation of all main LOX- and CYPmediated eicosanoids dominated by the CYP-mediated EETs and paralleled by lung injury [10]. In extension to these findings, the present work demonstrates that exposure of perfused human lungs to two different, naturally occuring bacterial agents, ECH and fMLP, induces 1) the generation of virtually all main lipid mediators of the AA cascade, LOXmediated LTs, COX-mediated PTs, CYP/sEH-mediated EETs/ 
DHETs, and HETEs mediated by LOX, COX or CYP, paralleled by 2) vasoconstriction and 3) oedema formation. In addition, it was noted that the AA/CYP/sEH-derived EETs/DHETs were, quantitatively, by far the dominant mediator group, apparently substantially contributing to the vasoconstrictor response, but not to the vascular leakage provoked by these agents. However, the LOX/COX-derived LTs/PTs were noted to make a major contribution to endothelial permeability increase, but caused only limited pulmonary vasoconstriction.

The two bacterial agents employed in the present study initiate eicosanoid generation via different mechanisms, which impose different requirements for endo-/exogenous free precursor AA availability, leading to differential effects on the kinetics of eicosanoid biosynthesis, such as the absolute dominance of eicosanoid release upon AA/fMLP coapplication over that upon ECH challenge. 1) The hydrophilic transmembrane poreforming ECH per se enables passive influx of extracellular $\mathrm{Ca}^{2+}$ and, thus, induces gradual phospholipase-dependent release of endogenous free precursor AA from cell membrane lipid pools $[17,39]$ by starting from zero and in limited amounts, which are readily converted by COX, LOX and CYP to equally limited amounts of eicosanoids. 2) In contrast to ECH, the receptor-operated ligand fMLP is not capable per se of major activation of endogenous AA-releasing phospholipases. Thus its capability to initiate the AA cascade is essentially dependent on the availability of exogenous free precursor AA $[16,43]$. As the whole amount of exogenously supplied free AA is available at once, before initiation of the AA cascade by bolus injection of $\mathrm{AMLP}$, in this case, the precursor is converted undelayed and quantitatively to eicosanoids, yielding higher maximal buffer concentrations than upon ECH challenge.

These different action mechanisms and AA availabilities may also explain the apparent lack of the $\mathrm{LTC}_{4}$ and $\mathrm{LTD}_{4}$ release patterns upon ECH challenge (fig. 2a) in contrast to the abundant liberation profiles of all cysteinyl-LTs upon coapplication of AA/fMLP (fig. 3a). 1) In the case of AA/fMLP co-application, due to the availability of the entire exogenously supplied precursor AA amount from the very beginning, not only the stable accumulating endproduct (characterised by progressive increasing and subsequent plateauing kinetics) $\mathrm{LTE}_{4}$, but also the short-lived intermediates (illustrated by fast peaking kinetics) $\mathrm{LTC}_{4}$ and $\mathrm{LTD}_{4}$ were generated in considerable and thus well detectable amounts (fig. 3a). 2) In contrast to $\mathrm{AA} / \mathrm{fMLP}$, upon ECH challenge, the endogenous precursor AA is provided gradually via phospholipase- and, thus, timedependent hydrolytic release from membrane lipids. Under these conditions of limited precursor availability, the fastpeaking intermediates $\mathrm{LTC}_{4}$ and $\mathrm{LTD}_{4}$ are rapidly further converted at much lower, not detectable concentrations, finally resulting in the stable accumulating end-product $\mathrm{LTE}_{4}$ present at higher and thus detectable concentrations (fig. 2a).

Of all eicosanoid classes, the LTs displayed the most pronounced dominance upon AA/fMLP versus ECH challenge by a factor of 10, compared with "only" 3, 1.8 and 2.6 for HETEs, PTs and EETs/DHETs, respectively. This may be explained by an additional amplification of the increase in LT generation due to exogenously supplied AA by multiple transcellular cooperative biosynthesis mechanisms typical for LTs, comprising a broad array of cell types, as described in the following. 1) The monitored LT profiles are compatible with the finding that even after extensive rinsing with buffer fluid, lungs harbour large, "resident" intracapillary pools of different leukocytes, but virtually no platelets $[14,15]$, and that the various resting pulmonary leukocyte populations (intravascular, interstitial and alveolar macrophages [44, 45]; lung mast cells [46]; intravascular granulocytes [3, 18]; monocytes [47]; and lymphocytes [48]) are known to be involved in the biosynthesis of LOX-derived lipid mediators. 2) The strong potency of the human lung vasculature for LT release in response to microbial challenge together with the lack $(<1 \%)$ of hydrolytic decay products of the unstable LT intermediate $\mathrm{LTA}_{4}$ is indicative of transcellular cooperative LT formation mechanisms. As LOX is restricted to cells of the myeloid lineage [6], transcellular LT synthesis is initialised in leukocytes where $\mathrm{LTA}_{4}$ is in part rapidly converted by $\mathrm{LTA}_{4}$ hydrolase to the chemotactic $\mathrm{LTB}_{4}$. The "surplus" $\mathrm{LTA}_{4}$, is released from donor leukocytes (PMNs, monocytes, macrophages, etc.) into the extracellular space, from where it is avidly taken up by other acceptor leukocytes, e.g. lymphocytes [49] or adjacent pulmonary acceptor ECs and converted to LTs, completely escaping extracellular degradation to the $\mathrm{LTA}_{4}$ decay products. The rapid conversion of $\mathrm{LTB}_{4}$ to the

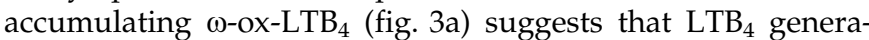
tion occured mainly in PMNs, the only cell type equipped with substantial $\omega$-oxygenase activity $[5,50]$. In contrast to LOX, the cysteinyl-LT synthesis-mediating glutathione-S-transferase leading to the generation of $\mathrm{LT}(\mathrm{C}, \mathrm{D}, \mathrm{E})_{4}$ occurs both in some myeloid cells, such as mast cells, eosinophils, basophils and monocytes/macrophages, as well as in ECs, but not in PMNs $[2,5,6,12]$. Consequently, cysteinyl-LTs are generated partly in cells equipped with both LOX and $\mathrm{LTC}_{4}$-synthase, such as mast cells and macrophages/monocytes [5, 12], but certainly mainly via transcellular cooperative synthesis. This includes an $\mathrm{LTA}_{4}$ shift from leukocyte donor cells to adjacent acceptor ECs followed by rapid conversion of $\mathrm{LTA}_{4}$ to cysteinyl-LTs [3, 18-21]. Transcellular cooperation in LT biosynthesis includes not only a shift of $\mathrm{LTA}_{4}$ from leukocyte donor to vascular acceptor cells with subsequent generation of LTs, but also release of free AA from activated ECs followed by uptake and processing of this precursor by adjacent leukocytes [16, 17].

The vasoregulatory and pro-/anti-inflammatory role of the HETEs in the pulmonary vasculature is largely unknown. Interestingly, continuous abundant liberation upon microbial challenge of the anti-inflammatory 15-HETE, previously isolated from human lung $[5,51]$, was noted. In contrast, only minor liberation of the proinflammatory 5-HETE was observed (table 1 and figs $2 b$ and $3 b$ ).

The vasodilatory PTs $\mathrm{PGI}_{2}$ (prostacyclin), $\mathrm{PGD}_{2}$ and $\mathrm{PGE}_{2}$ predominated quantitatively over the vasoconstrictive $\mathrm{PTs}$ $\mathrm{TXA}_{2}$ and $\mathrm{PGF}_{2 \alpha}$ (the levels of the strong vasoconstrictor TXA were 3.2-4.4 times lower than those of the potent vasodilator $\mathrm{PGI}_{2}$ ) (table 1 and figs $2 \mathrm{c}$ and $3 \mathrm{c}$ ), suggesting a rather moderate contribution of the vasoconstrictive PTs to the pressor response in the human lung. Although platelets are known as the main source of $\mathrm{TXA}_{2}$ biosynthesis, in contrast to resident leukocytes, the pool of marginated platelets in blood-free washed and perfused lungs is negligible [14]. Therefore, ECs and resident macrophages, monocytes, lymphocytes and PMNs, known as sites of extraplatelet $\mathrm{TXA}_{2}$ synthesis [52-54], may serve as 
alternative candidates for extraplatelet thromboxane generation. This may explain why even upon coapplication of AA/ $\mathrm{fMLP}$, i.e. availability of exogenous precursor $\mathrm{AA}, \mathrm{TXA}_{2}$ is the quantitatively absolute minor mediator (table 1).

The release of large amounts of CYP/sEH-mediated EETs and DHETs in the human lung vasculature upon microbial challenge is intriguing in view of the fact that little is yet known about their vasoregulatory role in the lung. Interestingly, the total amount of the LOX-mediated LTs, the LOX-, COX- or CYP-mediated HETEs, and the COX-mediated PTs, which are well known as mediators of pulmonary vasoconstriction, were dominated by a factor of three to four by that of the EETs/DHETs (fig. 4a, table 1). This absolute dominance of EETs and DHETs over the other lipid mediators of the AA cascade resembles that previously described in human lungs undergoing $\Delta P$ pa upon stimulation with $\mathrm{A} 23187$ [10] and considering the few available reports that describe the EETs and DHETs as vasoconstrictors in isolated, pressurised rabbit pulmonary arteries [24] and in the hypoxic rabbit and mouse lung $[31,38,55]$, the question arises of whether the high EET and DHET amounts may have an impact on vascular pressor response and/or oedema formation in human lungs upon microbial challenge.

Notably, simultaneous inhibition of LOX and COX in the AA/ fMLP-challenged human lung with MK886 and diclofenac, respectively, induced a pronounced decrease in LT and PT generation (fig. 4b) paralleled by marked reduction of weight gain (fig. 4c), suggesting a substantial contribution of LTs and PTs to oedema formation. Remarkably, only a limited attenuation of the human lung vascular pressor response to inflammatory challenge was registered (fig. 4c), although the generation of $\mathrm{LTB}_{4}$, cysteinyl-LTs, 5-HETE, vasoconstrictive PTs $\left(\mathrm{TXA}_{2}\right.$ and $\left.\mathrm{PGF}_{2 \alpha}\right)$ and vasodilatative PTs $\left(\mathrm{PGI}_{2}, \mathrm{PGD}_{2}\right.$ and $\mathrm{PGE}_{2}$ ) was strongly inhibited (to $12,23,15,21$ and $19 \%$, respectively, compared to AA/fMLP-challenge without LOX/ COX inhibition; fig. $4 \mathrm{~b}$ ), suggesting a possible contribution of the CYP/sEH-derived EETs and DHETs to pulmonary vasoconstriction.

Indeed, application of the selective [56] CYP epoxygenase inhibitor MS-PPOH prior to fMLP/AA challenge, though only undertaken in two experiments due to the shortage of human lungs available for the perfusion experiments, resulted in a considerable reduction of EET and DHET generation (fig. 4a) accompanied by an attenuation of the pressor response to $\sim 50 \%$, but not of oedema formation (fig. $4 \mathrm{c}$ ). These results correlate well with those obtained by exogenous application of 11,12-EET to human and rabbit lungs at the same final concentration as the $c$ max of endogenously released 11,12EET: induction of marked $\Delta P$ pa, but no significant $\Delta W$, although 11,12-EET and 8,9-EET, belonged to the quantitatively dominant regioisomers released in the human lung upon microbial challenge; thus, they are a potential prime candidate for causing oedema formation.

These findings are in agreement with reports [57, 58] describing a regiospecific, endothelial permeability-increasing activity of the four EET regioisomers in a concentration range of 3-10 $\mu \mathrm{M}$, i.e. surpassing by far the $\mathrm{cmax}$ of the EETs released in human lungs upon challenge with AA/fMLP $(0.04 \mu \mathrm{M}$
14,15-(EET+DHET), $0.56 \mu \mathrm{M}$ 8,9-(EET+DHET); concentrations in $\mu \mathrm{M}$ were converted from the respective values in $\mathrm{ng} \cdot \mathrm{mL}^{-1}$ in table 1), thus suggesting a rather negligible contribution of the EETs to oedema formation under these conditions.

Altogether, these data indicate that EETs generated in human lungs under conditions of challenge with bacterial agents elicit pronounced pressor response but no oedema formation.

In conclusion, stimulation of intact human lungs with two microbial agents, ECH and fMLP, elicited pulmonary vasoconstriction and vascular leakage, alongside the liberation of substantial amounts of all representative mediators of the AA cascade (LTs, HETEs, PTs and EETs/DHETs) into the perfusate. In response to both stimuli, the CYP/sEH-mediated EETs/DHETs clearly dominated over the sum of the other eicosanoid classes. Dual inhibition of LOX/COX in the AA/ fMLP-challenged human lung caused pronounced reduction of LT/PT biosynthesis and oedema formation but only limited attenuation of $\Delta P \mathrm{pa}$, while selective CYP epoxygenase inhibition induced marked suppression of EET/DHET generation and of the vasoconstrictor response but not attenuation of oedema formation, in accordance with exogenous EET application, which caused pulmonary arterial pressure rise but no lung weight gain. Next to well-known LOX- and COXmediated pulmonary vascular abnormalities, a major contribution of the CYP/sEH-mediated EETs/DHETs to the development of pulmonary pressor response may, thus, be expected.

\section{SUPPORT STATEMENT}

This work was supported by the Deutsche Forschungsgemeinschaft, Project KI 567/1-2 "Vaskuläre Funktionen Cytochrom P450-abgeleiteter Eicosanoide in der pulmonalen Strombahn", Project GR 1081/4 1 "Synthese und Metabolismus von Eicosanoiden in biologischen Systemen", Project KI 567/3-1 "Die Rolle von Cytochrom P450abgeleiteten Eicosanoiden in der Pathogenese der pulmonalen Hypertonie des Menschen", by a Research Grant of the University Medical Center Giessen and Marburg (UKGM) and by the Sonderforschungsbereich 547.

\section{STATEMENT OF INTEREST}

None declared.

\section{REFERENCES}

1 Needleman P, Turk J, Jakschik BA, et al. Arachidonic acid metabolism. Annu Rev Biochem 1986; 55: 69-102.

2 Henderson WR Jr. Eicosanoids and lung inflammation. Am Rev Respir Dis 1987; 135: 1176-1185.

3 Grimminger F, Becker G, Seeger W. High yield enzymatic conversion of intravascular leukotriene $\mathrm{A}_{4}$ in blood-free perfused lungs. J Immunol 1988; 141: 2431-2436.

4 Fitzpatrick FA, Murphy RC. Cytochrome P-450 metabolism of arachidonic acid: formation and biological actions of "epoxygenase"derived eicosanoids. Pharmacol Rev 1988; 40: 229-241.

5 Holtzman MJ. Arachidonic acid metabolism. Implications of biological chemistry for lung function and disease. Am Rev Respir Dis 1991; 143: 188-203.

6 Henderson WR Jr. The role of leukotrienes in inflammation. Ann Intern Med 1994; 121: 684-697.

7 Zeldin DC, Plitman JD, Kobayashi J, et al. The rabbit pulmonary cytochrome P450 arachidonic acid metabolic pathway: characterization and significance. J Clin Invest 1995; 95: 2150-2160. 
8 Grimminger F, Mayer K, Kiss L, et al. Synthesis of 4- and 5-series leukotrienes in the lung microvasculature challenged with Escherichia coli hemolysin: critical dependence on exogenous free fatty acid supply. Am J Respir Cell Mol Biol 1997; 16: 317-324.

9 Grimminger $\mathrm{F}$, Wahn $\mathrm{H}$, Mayer $\mathrm{K}$, et al. Impact of arachidonic versus eicosapentaenoic acid on exotoxin-induced lung vascular leakage: relation to 4-series versus 5-series leukotriene generation. Am J Respir Crit Care Med 1997; 155: 513-519.

10 Kiss L, Schütte H, Mayer K, et al. Synthesis of arachidonic acidderived lipoxygenase and cytochrome $\mathrm{P} 450$ products in the intact human lung vasculature. Am J Respir Crit Care Med 2000; 161: 1917-1923.

11 Roman RJ. P-450 metabolites of arachidonic acid in the control of cardiovascular function. Physiol Rev 2002; 82: 131-185.

12 Peters-Golden M, Henderson WR Jr. Leukotrienes. N Engl J Med 2007; 357: 1841-1854.

13 Palmer RM, Stepney RJ, Higgs GA, et al. Chemokinetic activity of arachidonic and lipoxygenase products on leukocytes of different species. Prostaglandins 1980; 20: 411-418.

14 Doerschuk CM, Downey GP, Doherty DE, et al. Leukocyte and platelet margination within microvasculature of rabbit lungs. J Appl Physiol 1990; 68: 1956-1961.

15 Ermert L, Duncker HR, Rosseau S, et al. Morphometric analysis of pulmonary intracapillary leukocyte pools in ex vivo-perfused rabbit lungs. Am J Physiol 1994; 267: L64-L70.

16 Grimminger F, Dürr U, Seeger W. Ligand-operated synthesis of 4series and 5-series leukotrienes in human neutrophils: critical dependence on exogenous free fatty acid supply. Mol Pharmacol 1992; 41: 757-766.

17 Grimminger F, Scholz C, Bhakdi S, et al. Subhemolitic doses of Escherichia coli hemolysin evoke large quantities of lipoxygenase products in human neutrophils. J Biol Chem 1991; 266: 14262-14269.

18 Grimminger F, Menger M, Becker G, et al. Potentiation of leukotriene production following sequestration of neutrophils in isolated lungs: indirect evidence for intercellular leukotriene A4 transfer. Blood 1988; 72: 1687-1692.

19 Grimminger F, Kreusler B, Schneider U, et al. Influence of microvascular adherence on neutrophil leukotriene generation. Evidence for cooperative eicosanoid synthesis. J Immunol 1990; 144: $1866-1872$.

20 Claesson HE, Haeggström J. Human endothelial cells stimulate leukotriene synthesis and convert granulocyte released leukotriene $\mathrm{A}_{4}$ into leukotrienes $\mathrm{B}_{4}, \mathrm{C}_{4}, \mathrm{D}_{4}$, and $\mathrm{E}_{4}$. Eur J Biochem 1988; 173: 93-100.

21 Feinmark SJ. Cooperative synthesis of leukotrienes by leukocytes and vascular cells. Ann N Y Acad Sci 1988; 524: 122-132.

22 Vanderhoek JY, Karmin MT, Ekborg SL. Endogenous hydroxyeicosatetraenoic acids stimulate the human polymorphonuclear leukocyte 15-LO pathway. J Biol Chem 1985; 260: 15482-15487.

23 Setty BNY, Stuart MJ. 15-hydroxy-5,8,11,13-eicosatetraenoic acid inhibits human vascular cyclooxygenase. J Clin Invest 1986; 77: 202-211.

24 Zhu D, Bousamra M II, Zeldin DC, et al. Epoxyeicosatrienoic acids constrict isolated pressurized rabbit pulmonary arteries. Am J Physiol Lung Cell Mol Physiol 2000; 278: L335-343.

25 Michaelis UR, Falck JR, Schmidt R, et al. Cytochrome P4502C9derived epoxyeicosatrienoic acids induce the expression of cyclooxygenase-2 in endothelial cells. Arterioscler Thromb Vasc Biol 2005; 25: 321-326.

26 Randriamboavonjy V, Kiss L, Falck JR, et al. The synthesis of 20HETE in small porcine coronary arteries antagonizes EDHFmediated relaxation. Cardiovasc Res 2005; 65: 487-494.

27 Campbell WB, Gebremedhin D, Pratt PF, et al. Identification of epoxyeicosatrienoic acids as endothelium-derived hyperpolarizing factors. Circ Res 1996; 78: 415-423.

28 Fisslthaler B, Popp R, Kiss L, et al. Cytochrome P450 2C is an EDHF synthase in coronary arteries. Nature 1999; 401: 493-497.
29 Busse R, Edwards G, Félétou M, et al. EDHF: bringing the concepts together. Trends Pharmacol Sci 2002; 23: 374-380.

30 Sandow SL. Factors, fiction and endothelium-derived hyperpolarizing factor. Clin Exp Pharmacol Physiol 2004; 31: 563-570.

31 Kiss L, Röder Y, Bier J, et al. Direct eicosanoid profiling of the hypoxic lung by comprehensive analysis via capillary liquid chromatography with dual online photodiode array and tandem mass-spectrometric detection. Anal Bioanal Chem 2008; 390: 697-714

32 Seeger W, Walter $\mathrm{H}$, Suttorp $\mathrm{N}$, et al. Thromboxane-mediated hypertension and vascular leakage evoked by low doses of Escherichia coli hemolysin in rabbit lungs. J Clin Invest 1989; 84: 220-227.

33 Seeger W, Walmrath D, Grimminger F, et al. Adult respiratory distress syndrome: model systems using isolated perfused rabbit lungs. Methods Enzymol 1994; 233: 549-584.

34 Kiss L, Bieniek E, Weissmann N, et al. Simultaneous analysis of 4and 5-series lipoxygenase and cytochrome P-450 products from different biological sources by reversed-phase high-performance liquid chromatographic technique. Anal Biochem 1998; 261: 16-28.

35 Garcia JGN, Noonan TC, Jubiz W, et al. Leukotrienes and the pulmonary microcirculation. Am Rev Respir Dis 1987; 136: 161-169.

36 Grimminger F, Walmrath D, Birkemeyer RG, et al. Leukotriene and hydroxyeicosatetraenoic acid generation elicited by low doses of Escherichia coli hemolysin in rabbit lungs. Infect Immun 1990; 58: 2659-2663.

37 Grimminger F, Thomas M, Obernitz R, et al. Inflammatory lipid mediator generation elicited by viable hemolysin-forming Escherichia coli in lung vasculature. J Exp Med 1990; 172: 1115-1125.

38 Pokreisz P, Fleming I, Kiss L, et al. Cytochrome P450 epoxygenase gene function in hypoxic pulmonary vasoconstriction and pulmonary vascular remodeling. Hypertension 2006; 47: 762-770.

39 Bhakdi S, Mackman N, Menestrina G, et al. The hemolysin of Escherichia coli. Eur J Epidemiol 1988; 4: 135-143.

40 Voelkel NF, Czartolomna J, Simpson J, et al. FMLP causes eicosanoid-dependent vasoconstriction and edema in lungs from endotoxin-primed rats. Am Rev Respir Dis 1992; 145: 701-711.

41 Abernathy VJ, Webster RO, Dahms TE. C-reactive protein inhibits increased pulmonary vascular permeability induced by fMLP in isolated rabbit lungs. Am J Physiol 1996; 271: H507-H513.

42 Tanaka H, Bradley JD, Baudendistel LJ, et al. Mechanisms of increased pulmonary microvascular permeability induced by FMLP in isolated rabbit lungs. J Appl Physiol 1992; 73: 2074-2082.

43 Palmer RMJ, Salmon JA. Release of leukotriene B4 from human neutrophils and its relationship to degranulation induced by $\mathrm{N}$ formyl-methionyl-leucyl-phenylalanine, serum-treated zymosan and the ionophore A23187. Immunology 1983; 50: 65-73.

44 Bertram TA, Overby LH, Danilowicz R, et al. Pulmonary intravascular macrophages metabolize arachidonic acid in vitro. Am Rev Respir Dis 1988; 138: 936-944.

45 Rose F, Kiss L, Grimminger F, et al. E. coli hemolysin-induced lipid mediator metabolism in alveolar macrophages: impact of eicosapentaenoic acid. Am J Physiol Lung Cell Mol Physiol 2000; 279: L100-L109.

46 Samuelsson B, Dahlen SE, Lindgren JA, et al. Leukotrienes and lipoxins: structures, biosynthesis, and biological effects. Science 1987; 237: 1171-1176.

47 Juergens UR, Christiansen SC, Stevenson DD, et al. Inhibition of monocyte leukotriene $\mathrm{B} 4$ production after aspirin desensitization. J Allergy Clin Immunol 1995; 96: 148-156.

48 Jakobsson PJ, Shaskin P, Larsson P, et al. Studies on the regulation and localization of 5-lipoxygenase in human B-lymphocytes. Eur J Biochem 1995; 232: 37-46.

49 Jakobsson PJ, Odlander B, Claesson HE. Effects of monocytelymphocyte interaction on the synthesis of leukotriene $\mathrm{B}_{4}$. Eur $\mathrm{J}$ Biochem 1991; 196: 395-400.

50 Nadeau M, Fruteau de Laclos B, Picard S, et al. Studies on leukotriene $\mathrm{B}_{4} \omega$-oxidation in human leukocytes. Can J Biochem Cell Biol 1984; 62: 1321-1326. 
51 Hunter JA, Finkbeiner WE, Nadel JA, et al. Predominant generation of 15-lipoxygenase metabolites of arachidonic acid by epithelial cells from human trachea. Proc Natl Acad Sci USA 1985; 82: 4633-4637.

52 Ingerman-Wojenski C, Silver MJ, Smith JB, et al. Bovine endothelial cells in culture produce thromboxane as well as prostacyclin. J Clin Invest 1981; 67: 1292-1296.

53 Nüsing R, Lesch R, Ullrich V. Immunohistochemical localization of thromboxane synthase in human tissues. Eicosanoids 1990; 3: 53-58.

54 Bizios R, Lai L, Fenton JW 2nd, et al. Thrombin-induced thromboxane generation by neutrophils and lymphocytes: dependence on enzymic site. J Cell Physiol 1987; 132: 359-62.

55 Keserü B, Barbosa-Sicard E, Popp R, et al. Epoxyeicosatrienoic acids and the soluble epoxide hydrolase are determinants of pulmonary artery pressure and the acute hypoxic pulmonary vasoconstrictor response. FASEB J 2008; 22: 4306-4315.

56 Wang MH, Brand-Schieber E, Zand BA, et al. Cytochrome P450derived arachidonic acid metabolism in the rat kidney: characterization of selective inhibitors. J Pharmacol Exp Ther 1998; 284: 966-973.

57 Alvarez DF, Gjerde EAB, Townsley MI. Role of EETs in regulation of endothelial permeability in rat lung. Am J Physiol Lung Cell Mol Physiol 2004; 286: 445-451.

58 Ivey CL, Stephenson AH, Townsley MI. Involvement of cytochrome P-450 enzyme activity in the control of microvascular permeability in canine lung. Am J Physiol Lung Cell Mol Physiol 1998; 275: L756-L763. 